\title{
On the Futility of Contact Tracing
}

\author{
Jay Bhattacharya \&t Mikko Packalen
}

$\mathrm{T}$

HROUGHOUt THE COVID-19 epidemic, public health authorities have promoted contact tracing as a key tool to combat the spread of the SARS-CoV-2 virus. Nearly every country infected by the virus has adopted some version, though with evidently mixed results given the global spread of the epidemic. Our purpose in this essay is to make the case that, contrary to conventional public health wisdom, most of the hope placed on contact tracing efforts to control the epidemic is ultimately futile. It may be useful when the number of cases in an epidemic is very small and only if it is applied aggressively without regard to privacy rights. In cases that do not fit that description, contact tracing may make an outbreak worse.

\section{$\mathrm{T}$}

HE IDEA BEHIND classic contact tracing is simple and intuitive. Suppose that public health officials can identify an index case of an infectious disease-by definition, the first documented patient. That patient must have been infected by someone else: the disease is contagious, after all. And that person must have been infected by someone else. Classic contact tracing is the systematic application of the idea of working recursively backward to trace the disease back to a source. All those identified along the way who are still infected can either be quarantined or treated (if there is a treatment) so that they do not further spread the disease. This form of contact tracing is used routinely for the control of venereal diseases. It is most effective when the infection would not naturally resolve itself over a period shorter than the time needed to track backward to patient zero.

A variant form of contact tracing, the one actually in use for COVID-19, works forward instead. Suppose that an index patient tests positive for COVID-19. In this variant, the contact tracer will ask the patient to report all the people with whom he has been in contact, and all the places where contact took place. The tracer will then work through the list of contacts, presumptively mandating a two-week quarantine unless a polymerase chain reaction (PCR) test, if available, shows the contact to be uninfected. The quarantine is maintained while the PCR test result comes back, which may take several days or more. In many cases, especially early in the epidemic, the PCR test was not available, so the two-week quarantine was presumptively enforced. The tracing exercise works recursively forward, identifying contacts in concentric circles of contacts on the idea that the index person may have spread the virus to them. Any contact who traces positive is also subject to the same contact tracing exercise.

The appeal of this approach is the same as the one provided by looking for one's lost keys under the glow of a streetlight. The keys may not be there, but there is little hope of finding them anywhere else. COVID-19 is an infectious disease, so tracing and testing contacts focuses the attention of public health officials on a set of people who are more likely than a randomly chosen individual to have the disease. Since there is no effective treatment that hastens the resolution of the infection, quarantining is the only way to prevent potentially infected individuals from spreading the disease.

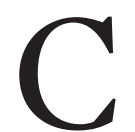

ONTACT TRACING HAS A long and checkered history and was in use even before the germ theory of disease. In sixteenth-century Malta during a plague epidemic, city authorities quarantined newly arriving sailors, forcing them into lazarettos (plague hospitals). ${ }^{1}$ The physicians treating the sailors were isolated, even if they showed no symptoms, since they were in close contact with the sailors. Since Yersinia pestis, a bacterium which infects fleas and is carried by rats, causes the plague, the quarantine could not have slowed the spread of the disease, and, indeed, may have intensified the epidemic given the poor sanitary conditions of the lazarettos.

Eugenia Tognotti has described an early version of the techniques used to control a cholera outbreak in Europe during the nineteenth century:

In cities, authorities adopted social interventions and the traditional health tools. For example, travelers who had contact with infected persons or who came from a place where cholera was present were quarantined, and sick persons were forced into lazarettos. In general, local authorities tried to keep marginalized members of the population away from the cities. ${ }^{2}$ 
As with the plague, contact tracing in this context likely had little effect on the spread of cholera-a disease caused by fecal contamination of the water supply-and may have served to prolong the epidemic because of poor sanitary conditions in the quarantine hospitals.

By contrast, public health used contact tracing with considerable success in the conquest of smallpox in the late twentieth century. ${ }^{3}$ After the development of an effective smallpox vaccine, the World Health Organization in 1959 developed a plan to eradicate the disease through worldwide mass vaccination. By the mid-1970s, the campaign had worked to sharply limit the spread of the virus, but there were still occasional outbreaks in places where there were unvaccinated people. Whenever authorities became aware of a case, they would quarantine the index patient and initiate a campaign to vaccinate every person within the vicinity who may have been exposed. Contact tracing worked to help eradicate the disease because, by the mid-1970s, the vaccine had limited the epidemic to a few localized, disconnected outbreaks. Of course, there is no known effective SARS-CoV-2 vaccine, and the disease is widespread and global. The smallpox example provides no support for contact tracing as a tool for disease eradication in the case of COVID-19.

I $\mathrm{N}$ WHAT FOLLOWS, we argue first that the epidemic is too widespread for contact tracing to limit disease spread; second, that errors in PCR tests substantially raise the human costs of contact tracing and render it less effective; and finally, that contact tracing creates strong incentives among the public to mislead public health authorities and avoid voluntary testing.

COVID-19 infection is already too widespread in the United States, and many other countries, for contact tracing to work effectively. Seroprevalence studies of COVID-19 antibodies provide definitive evidence. The presence of a specific antibody in any given individual provides strong evidence of prior infection by SARS-CoV-2. To date, scientists around the world have conducted over 50 population-level seroprevalence studies that all point to the same qualitative conclusion. ${ }^{4}$ The COVID-19 epidemic is between 5 and 400 times more widespread than case reports, which detect active infections, might indicate. For example, although case reports in the United States suggest there were just over 1 million identified cases by late April 2020, ${ }^{5}$ a recent study by the Centers for Disease Control and Prevention found evidence that there were between 6 and 24 times that many infections. ${ }^{6}$

The seroprevalence evidence establishes a crucial fact: a large fraction-as much as half, or more-of all people infected by SARS-CoV-2 never develop symptoms at all, or at worst, develop mild cold-like symptoms. ${ }^{7}$ While people who have no symptoms are less likely to spread the disease than people who expectorate virus-filled droplets, there is good evidence that the asymptomatic infected can spread the disease. ${ }^{8}$ Since there are so many COVID-19 patients with no symptoms, it should be no surprise that the asymptomatic infected, in aggregate, are responsible for many new cases.

There is an immediate corollary. Even in places that test populations aggressively for an active infection, a large fraction of COVID-19 cases are never identified. The exact multiple of unidentified to identified active cases varies from place to place. Where PCR testing is scarce, the multiplier will be larger. Clinical and policy decisions about selection for PCR testing also contribute to this multiple. The point should be clear: many people get this disease who never come to the attention of public health authorities.

These facts, taken together, undermine the efficacy of contact tracing. The sheer scope of cases means that the scale of a contact tracing effort would have to be monumental to appreciably slow disease spread. Recursive forward tracing would eventually encompass the whole population. This fact explains why proposals for contact tracing in the US typically feature an army of thousands of contact tracers-a smaller effort would make little difference to the epidemic. ${ }^{9}$ What is more, the presence of so many asymptomatic people spreading the disease sub rosa means that contact tracing cannot work-the strategy will never identify transmission stemming from unidentified, asymptomatic cases. While a contact tracing strategy may lead by chance to the identification of these cases, there is no reason to expect that the practice will systematically identify all, or even most, of them. The disease will continue to spread despite the contact tracing policy.

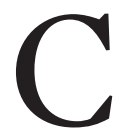

ONTACT TRACING EFFORTS rely on an accurate test for the virus with rapidly available results to avoid the arbitrary application of quarantines. Unfortunately, this description is only applicable in part to the most widely used test to diagnose cases of COVID-19. Reverse transcription polymerase chain reaction (RT-PCR) tests check for active SARS-CoV-2 infection by amplifying genetic material that is specific to SARS-CoV-2. The tests are widely seen in medicine and public health as the gold standard test for active infection. The return of a PCR testing result can take between 24 hours and several days.

Though the RT-PCR test is very accurate, like any medical test, it does have errors. Early in the course of infection, the test may return a negative result among patients who have an active infection. False-negative results may also occur because the test requires an unpleasant nasal swab. Testers will sometimes probe less deeply than is necessary to collect an appropriate sample. The possibility of false negatives in PCR testing means that contact tracing will miss cases, prematurely ending the forward recursion before it reaches its ultimate end. One (not yet peer-reviewed) study estimates a 33\% false-negative rate from a single PCR test conducted only ten days after symptom 
onset. ${ }^{10}$ Another study (this one peer-reviewed) finds an even higher false-negative rate in the days before symptom onset. ${ }^{11}$ Contact tracing supported by a test with a substantial proportion of false negatives will be less efficient in achieving disease mitigation than it would if a more accurate test supported it.

PCR testing also has a functional false-positive problem. The test is very specific to the SARS-CoV-2 virus in that other viruses or other genetic material will not falsely cause a positive test result. Nevertheless, PCR tests often return a positive result for some time after COVID-19 patients have cleared their infection. Several studies find that recovered patients retain nonviable viral fragments shattered by the patients' immune system. ${ }^{12}$

In addition, PCR tests for SARS-CoV-2 are often tuned to be too sensitive, registering a positive test result even when the amount of virus present is non-infectious. Before running a PCR test, clinical laboratories must decide how many doubling cycles are needed before a sample can be declared positive or negative. If too many doubling cycles are required to amplify the viral genetic material before it can be detected, the patient does not have enough virus present to be infectious. ${ }^{13}$ Since these patients do not pose any risk to others but still retain the viral genetic material, the PCR test result is a functional false positive.

In principle, one may think that the availability of testing mitigates some of the costs of being identified as a contact-an extended quarantine is imposed only if the contact tests PCR positive. The fact that false positives are possible means that premising the quarantine on a positive test may not reduce the costs of contact tracing on identified contacts as much as one may suppose.

Or hope.

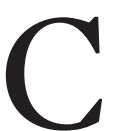

ONTACT TRACING REQUIRES infected individuals under the scrutiny of contact tracers to act against their own interests. Though the questions that contact tracers ask infected patients may seem benign, tracers are asking them to divulge sensitive or private information. While it may be easy for a COVID-19 patient to tell their interviewer that they went grocery shopping, it may be much harder to admit that they attended a private dinner party. Admitting to an illicit tryst would be out of the question. Even less embarrassing admissions can place a patient's friends at the center of an unwanted investigation. And since there is no effective treatment or cure for COVID-19, there is no offsetting personal benefit gained to the friend from the unwanted referral-just the risk of an unplanned multiple-day quarantine until the SARS-CoV-2 PCR test result comes back. Contact tracers themselves may limit what they ask patients for a variety of reasons, including the political sensitivity of the questions. There are, for example, reports that some contact tracers in New York City will not ask patients about their participation in protests. ${ }^{14}$ This is not just a theoretical problem. In Los
Angeles, for instance, $60 \%$ of positive patients refuse to provide any information about their contacts to the tracers. ${ }^{15}$

Incomplete reporting of the sort described would diminish the efficiency of any contact tracing program. One might reason, however, that this is not a fatal flaw; as long as there is enough overlap in the physical social networks of the infected population, an aggressive contact tracing regime would eventually identify all cases even if some patients were not entirely forthcoming or cooperative. Unfortunately, this reasoning ignores a basic economic fact about the incentive to get tested.

Under a contact tracing regime, getting tested for active COVID-19 infection is no longer an uncomplicated clinical decision aimed at providing a physician with information about how to manage a patient. Contact tracing adds the additional cost of having to put friends and coworkers at risk of quarantine if the PCR test is positive. While there is a clear public benefit from identifying and isolating positive cases, individual patients gain little or no private benefit. At the very least, even very public-minded patients will not capture the full public benefit, which would accrue to other people. Contact tracing thus introduces a disincentive for people who believe they might be SARS-CoV-2 positive to be tested in the first place.

\section{$\mathrm{T}$}

HERE are INSTANCES where contact tracing strategies have been apparently successful. A comparison of the experiences of China, South Korea, and Germany with contact tracing is instructive about the circumstances of these successes.

China implemented a draconian contact tracing and quarantining regimen that reduced the time for public health authorities to identify new cases from 4.6 days after developing symptoms to 2.7 days. ${ }^{16}$ Chinese authorities mandated that its citizens download a cell phone app to track the movements, credit card use, and other information of the population of affected regions in support of its contact tracing program. As of late September, there have been 59 identified cases and three deaths per million people in China. ${ }^{17}$

The South Korean contact tracing program ${ }^{18}$ is credited with limiting the outbreak in that country. ${ }^{19}$ It provides publicly available access to detailed information about the age, sex, neighborhood of residence, and travel history of index cases. Understandably, COVID-19 patients fear the stigma of public identification. As of late September, there have been 453 identified cases and eight deaths per million people. ${ }^{20}$

Germany hired 16,000 contact tracers and mandated 14-day quarantines for all contacts who spent at least 15 minutes in the presence of the index patient within the previous two days. ${ }^{21}$ German authorities contemplated mandating a Chinese-style contact tracing app but ultimately decided against it because of privacy concerns. As of late September, there have been 3,314 identified cases 
and 113 deaths per million people; case numbers have been rising recently as Germany has relaxed its lockdown. ${ }^{22}$

The immediate and obvious lesson to draw from this comparison is that aggressive contact tracing can and has worked to slow the spread of COVID-19 in some countries. But one should not be too hasty in drawing this conclusion, as there is a plausible alternate hypothesis. A study published recently in Cell identified a mutation that increases the infectious potential of the virus by 10 -fold over the original Wuhan-1 strain. ${ }^{23}$ The hypothesis supported by this study is that, while the less infectious Wuhan-1 strain dominated the Chinese and South Korean outbreaks, the more infectious variant dominated the German epidemic, explaining the larger number of cases. Contact tracing probably played a role in limiting disease spread, but given this plausible alternate hypothesis, the full extent of its role is not yet clear.

Another lesson to learn from this comparison is that, if contact tracing is to work for COVID-19, it must be very aggressively applied with little regard for the privacy rights of individuals. A third lesson is one we have already learned, which is that contact tracing is most successful very early on in the epidemic, and less effective later when the disease has already spread widely.

A final lesson, one hidden by the approach of comparing the experience of various countries: even a successful contact tracing effort leaves most of the population vulnerable to future infection by SARS-CoV-2. The world is interconnected, and the virus is still present in the population of every continent. No country can isolate itself forever. SARS-CoV-2 remains an incredibly infectious disease. Countries that have controlled the epidemic early through contact tracing programs will have to continue to quarantine foreign visitors and keep in place intrusive contact tracing measures indefinitely to avoid a future spread of the virus through their population. Countries that choose this path will face enormous economic costs as they become increasingly disconnected from the systems of global trade.

I S CONTACT TRACING now futile for the COVID-19 epidemic in most of the world? Probably yes. The infection is already too widespread for disease eradication to be feasible in most places. It might be useful in slowing the spread of the epidemic in areas where hospital resources are at risk of congestion. This benefit will come, however, at considerable costs to the privacy rights and civil liberties of traced individuals, often with no public health benefit because of testing errors. Some may argue that these costs are worth paying temporarily if contact tracing really can help slow the spread of the epidemic. But contact tracing creates incentives to avoid testing and may make the epidemic worse. Studies involving contact tracing can provide useful information to help us better understand transmission dynamics-one fascinating study involving contact tracing has clarified the very limited role that children play in disease spread. ${ }^{24}$ Apart from this ancillary benefit, contact tracing does not deserve the central place it has received in the tool kit public health authorities use to control COVID-19.

\section{Jay Bhattacharya is a Professor of Medicine at Stanford University and a Research Associate at the National Bureau of Economic Research.}

\section{Mikko Packalen is an Associate Professor of Economics at the University of Waterloo.}

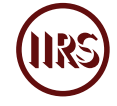

1. Monique Grech, “The Order of St. John's Approach to Plague after Rhodes," Melita Historica 16, no. 2 (2013): 35-44.

2. Eugenia Tognotti, "Lessons from the History of Quarantine, from Plague to Influenza A," Emerging Infectious Diseases 19, no. 2 (2013): 256, doi:10.3201/eid1902.120312.

3. "History of Smallpox," US Centers for Disease Control and Prevention, August 30, 2016.

4. John Ioannidis, "The Infection Fatality Rate of COVID-19 Inferred from Seroprevalence Data," medRxiv (2020), doi:10. 1101/2020.05.13.20101253.

5. “CDC COVID Data Tracker,” US Centers for Disease Control and Prevention.

6. Fiona Havers et al., "Seroprevalence of Antibodies to SARSCoV-2 in 10 Sites in the United States, March 23-May 12, 2020," JAMA Internal Medicine (2020), doi:10.1001/jamainternmed.2020.4130.

7. Maryam Shakiba et al. "Seroprevalence of COVID-19 Virus Infection in Guilan Province, Iran," medRxiv (2020), doi:10.1 101/2020.04.26.20079244; Lisa Mullins and Lynn Jolicoeur, “Testing Reveals 'Stunning' Asymptomatic Coronavirus Spread among Boston's Homeless," WBUR, April 14, 2020; and Hiroshi Nishiura et al., "Estimation of the Asymptomatic Ratio of Novel Coronavirus Infections (COVID-19)," International Journal of Infectious Disease 94 (2020): 15455, doi:10.1016/j.ijid.2020.03.020.

8. Hanalise Huff and Avantika Singh, "Asymptomatic Transmission during the Coronavirus Diesase 2019 Pandemic and Implications for Public Health Strategies," Clinical Infectious Diseases (2020), doi:10.1093/cid/ciaa654.

9. "California To Hire Thousands of Contact Tracers As Part of Reopening Plan," CBS Sacramento, May 5, 2020.

10. Paul Wikramaratna et al., "Estimating False-Negative Detection Rate of SARS-CoV-2 by RT-PCR," medRxiv (2020), doi:1 0.1101/2020.04.05.20053355.

11. Lauren Kucirka et al., "Variation in False-Negative Rate of Reverse Transcriptase Polymerase Chain Reaction-Based SARS-CoV-2 Tests by Time Since Exposure," Annals of Internal Medicine (2020), doi:10.7326/M20-1495. 
12. Lan Lan et al., "Positive RT-PCR Test Results in Patients Recovered from COVID-19," JAMA 323, no. 15 (2020): 1,502-503, doi:10.1001/jama.2020.2783; Andrew Cohen, Bruce Kessel, andMichael Milgroom, “Diagnosing COVID19 Infection: the Danger of Over-Reliance on Positive Test Results," medRxiv (2020), doi:10.1101/2020.04.26.20080911; Yasemin Saplakoglu, "Recovered Patients Who Tested Positive for COVID-19 Likely Not Reinfected," livescience.com, April 30, 2020; and Alireza Tahamtan and Abdollah Ardebili, "Real-Time RT-PCR in COVID-19 Detection: Issues Affecting the Results," Expert Review of Molecular Diagnostics 20, no. 5 (2020): 453-4, doi:10.1080/14737159.2020. 1757437.

13. Tom Jefferson et al., "Viral Cultures for COVID-19 Infectivity Assessment. Systematic Review," medRxiv (2020), doi:10 $.1101 / 2020.08 .04 .20167932$.

14. Greg Smith, "NYC COVID-19 Contact Tracers Not Asking about George Floyd Protest Participation, Despite Fears of New Virus Wave,” The City, June 14, 2020.

15. Melody Petersen, "LA County's Tracing Team Repeatedly Failed to Detect Coronavirus Outbreaks at Workplaces," Los Angeles Times, July 31, 2020.

16. Qifang Bi et al., "Epidemiology and Transmission of COVID-
19 in 391 Cases and 1,286 of Their Close Contacts in Shenzhen, China: A Retrospective Cohort Study," Lancet Infectious Diseases 2, no. 8 (2020): 911-9, doi:10.1016/S1473-3099(20) 30287-5; and Victor Tangermann, "In China, This Coronavirus App Pretty Much Controls Your Life," Futurism, April $16,2020$.

17. “Coronavirus Statistics Global," Epidemic-stats.com (2020).

18. Max Kim, "Seoul's Radical Experiment in Digital Contact Tracing," New Yorker, April 17, 2020.

19. Alexandra Sternlicht, "South Korea's Widespread Testing and Contact Tracing Lead to First Day with No New Cases," Forbes, April 30, 2020.

20. "Coronavirus Statistics Global."

21. Loveday Morris and Luisa Beck, "While US Struggles to Roll out Coronavirus Contact Tracing, Germany Has Been Doing It from the Start," Washington Post, May 25, 2020.

22. "Coronavirus Statistics Global."

23. Qianqian Li et al., "The Impact of Mutations in SARS-CoV-2 Spike on Viral Infectivity and Antigenicity," Cell 182, no. 5 (2020): 1,284-94, doi:10.1016/j.cell.2020.07.012.

24. Daniel Gudbjartsson et al., "Spread of SARS-CoV-2 in the Icelandic Population," New England Journal of Medicine 382 (2020): 2,302-15, doi:10.1056/NEJMoa2006100. 